\title{
Discussion on Functional Division for Landscape Design Teaching
}

\author{
Lei Cai \\ Yunnan Institute of Technology and Information \\ Kunming, China 650224
}

\author{
Shifang Xu \\ Kunming Panlong District Planning Bureau \\ Kunming, China 650224
}

\begin{abstract}
This article discusses the functional division that is used generally in the teaching of landscape design, and it is not to give a yes or no simply but makes analysis from the development of the era and cross disciplines, trying to avoid philosophical arguments but unable to get basis out of the philosophy. The author draw a conclusion at the end of the article, characteristics of the landscape are dynamic and open, to some considerations are necessary for the design. Function can not be divided mechanically, instead, attentions shall be paid to laws of activities of the people in space and time and rational arrangements shall be made to complete the task.
\end{abstract}

Keywords-landscape design; professional teaching; function; division

\section{INTRODUCTION}

During the teaching of landscape design, the author often encounters functional division, which requires students to make functional analysis in a design, traditionally the students will draw a functional division diagram according to the habitual thought, expressing via bubble diagram and adding bubbles on the completed landscape plan, then rack their brains to fill names in the bubbles such as children activity area, waterscape area, mountain forest area, sports area and so on, even some just take the functional division diagrams as a task to increase the drawing thickness. Students often question: Is the functional drawing necessary? When is the functional division needed? Doest the functional division diagram cover all areas? If necessary, How is the space treated? It is hard to get a correct name for the functional divisions. How can correct and simple name be got?

Facing so many questions from students, the author, as a professional teacher of landscape, is promoted to think how to clearly explain "the landscape design thought and expression", namely the functional division. It is difficult to seek when the functional division diagram is used in the landscape design, yet it has been widely known that for a long time, the development of landscape design thought is greatly influenced by that of the architectural design and urban planning, this article is not to research how it is influenced or how to build and plan, instead it aims at two problems: Is the landscape design needed for functional division; How is it achieved?

\section{EXPLANATION OF FUNCTION AND DIVISION}

First of all, it needs to divide the function division into two parts for analysis, seen from natural philosophy, it is unable to bind an object together with functions, this is, it is not said every natural object has functions of its own or its functions are not discovered, the function is recognition of the people to an object. Darwin ever described the relations between function and form from "survival of the fittest" and "natural selection", a function is not owned when an object is born, it is gradually form during the mutual influence with the environment and disappearing when the object is gone or changed into something in other forms. The combination of function and object can be reflected in many aspects, some are reflected by elegant and beautiful appearance in order to aesthetics; some are reflected via living functions accommodated in an interior space; some are reflected by characteristics of materials, for example, the transparency of glass make it easy for lighting; some are reflected on the purpose of its own, for example, a kettle to boil water, even it looks ugly, it still can be used to boil water. The functions mentioned may exist in an object independently or coexist. The recognition of human being to an object brings function, namely purpose, any function responds to certain purposes. In the opinion of Vitruvius, purposes of architecture and landscape are solid, practical and delighted, the function of ancient classic landscape changed from communication, sacrifice (metal function) to garden (production and breeding), palace court yard (enjoyed from governors, literati and officialdom and merchants and to public gardens (amusement by the public and participant), whose evolution shows the goal-oriented characteristics.

Philosopher Nietzsche said, seed is something real and a treasure of personalities, and the will to rights is hidden in the elaborate mechanism, and its function is just to discover the prefect performance of itself in the form. Guided by Whitman, Darwin and Spinoza and enlightened by Philosopher Nietzsche, Louis Sullivan took architecture as the scatter of vitality, so the perfect architectural form and function that functionalists hold at heart are closely connected, and a thought "Forms follows Function" came in to being. With the time going, the concept has gradually become a standard for modernists to judge wrong from right as a whole. [1] The thought "Forms follow Function" thoroughly overturned "the one and only way" that the school of Ecole nationale supérieure des beaux-arts de Paris held, which find a solid way for the development and design of modern architecture after the Industrial Revolution and the 
huge changes in systems, society, economy and industry. However, the old generation of architects who advocated functions failed to continue the theory of architectural functions, instead they were restricted by mechanical functions just because of the absolution and abstraction of function concepts. After the functionalism of architecture are wildly questioned, a generalized functional theory becomes popular, which thinks that with the more and more architectural functions developed such as use function, spiritual function, urban beautification, social politics and so on, the people are enlightened to think of the architecture from a wide scope. Yet some scholars (as Han Dongqing, 1996) though that the functional theory is too inclusive, as such, any era, school or claims in the history of architecture almost can be integrated to the functionalism, it is hard to say how it work to clarify the history and instruct the practice, which even help the regeneration of formalism. Han Dongqing said, seen from the nature, architectural function refers to activities of the people supported by architecture as well as the activity nature, mutual relation, laws of changes, the real purpose of architecture is the life of the people. For the life is dynamic, architectural function shall be dynamic as well. In the meantime, he discussed the dynamics of architectural functions from four aspects as inclusiveness, compatibility, individual explanation and weeks, and the dynamic characteristics require that the architectural design must be connected with the society, the city and the people's life so as to expand the true meaning of the function. We should not only regard the obvious function of architectures but also recognize the potential function of the architecture in the history [2].

"Division" means the tangible space and site will be divided for purposes as per given function and need, which will be used to organize and deal with the mutual relations between spaces and sites and also solve many contradictions and questions of reality. Le Corbusier, modernism architect, in the stage of rational architecture claimed that a city shall be rational and highly functional the same as architecture. In the Brilliant City and Tomorrow's City, Le Corbusier stressed four functions for the rational development of a city, namely residence, living, work and traffic, traffic lines connect the areas altogether to form a whole body, which directly caused the direction for the compilation of Athens Charter on CIAM in 1933. According to the Athens Charter, the urban space is a division of the urban function, the city is divided into different functional areas, then convenient traffic network is used to connect the functional areas. During the industrial development, in the unceasingly expanded medium and large cities, industrial areas were mixed with residential areas, the industrial pollutions were much serious, the lands were highly used, there were no supporting facilities, lack of empty sites and the traffic jam had caused serious problems in health, traffic and residence. Seen from the point, the application of functional division may surely solve the real problems existing in cities, improve the cities out of order so as to make cities meet the basic needs of most people in psychology and physiology, having some significance in practice and history. Therefore, in the urban planning after the World War II, the functional division, as the most basic principle for the urban space organization, has been widely applied and practiced. However, due to the overstressing of pure functional division in practice, a series of problems have arisen, which seriously damaged the urban planning, yet it's the fault of the principle itself. In 1952, Jane Jacobs released her works The Death and Life of Great American Cities which criticized the "pure and sterile", "mechanically rational" pure urban functional division theory led by Le Corbusier, in her opinions, such planning only causes the loss of urban energy and diversity [3]. Accordingly the division itself is not wrong, it may adjust and make the disordered sites be in order. Yet with the time going, the cities begin to take on diversity, even the scenes and challenges faced by the same landscape design become much more complicated, so the pure landscape function is unfeasible.

\section{ANALYSIS OF DIFFICULTIES FOR THE FUNCTIONAL DIVISION OF LANDSCAPE}

Functional division drawings become more and more difficult to draw, one of the main reasons is, due to the development of the era and changes of social psychology and ideology, the function of landscapes and the need of the people to them have changed as well. Drawings are media to express design thought and construction instruction, each of which contains different information and intension of expression, and the functional division diagrams main try to indicate the function type of lands in the design and corresponding relations with the site. Yet for the landscape is functionalized in an open space, which is not the same as the architectural design and urban planning where walls and land redlines are used to mark the boundaries of functions, so the functional division is difficult to achieve. In addition, the functional connotation of landscapes is much richer than architecture, and it takes site, space and facilities as carriers and the users may enjoy diverse functions indoors or outdoors such as break, relaxation, swimming, chatting, visit, running, picnic, scientific education and so on, whose historical time length will be shorter than architecture, relevant content change frequency and complication are higher as well. In addition, functional division diagrams are an expression on the plan, yet with the time going and the deeper understanding of the people in landscapes, 3D and 4D landscapes are stressed, and the expression on single plans is unable to clearly show the laws of "functional activity stream" and "conscious stream", which may cause many problems for the following construction and use in practice.

For a long time, the landscape design stresses and follows three principles "applicable, economic and beautiful", which are dialectical and unified, mutually inter-dependant and indivisible. "Applicable" is not equal to "practicable", it is dynamic to some degree, reflecting the adaptation of the landscape design to the diversity in type, region and scale, based on which, the functions will be arranged. In the methodology it is called "site analysis", through the on-the-spot investigation, research and analysis, it needs to grasp the project land scale, ground creature and regional culture before the functional division and factors are distributed, which are basic for foundation. On the other hand, it shall be abundant reflected in the space, actually the activities of the people will not occupy all spaces, there must be considerable abundance between users and the space[2]. In fact, what the landscapes face are functional activities which are abundant and highly different, having a high requirements on the abundant space. 
Garrett Eckbo, a core scholar of Californian school, adopted flexible vegetation and low walls to separate and arrange the space after absorbing the flowing space thought of Mies, creating a abundant space system with flow and inclusiveness.

Whether in the East or the West, starting from the beginning, the landscape has some purposes and compliance. Over thousands of years, we have been making use of materials such as natural environment, water, soil, stone, plant, animal and structures to create places for break and landscapes, whose main functions include amusement and break, beautifying the environment and improving biology[4]. Going with the time, the mutual effect and relations between modern landscapes and environment and human being are becoming more and more complicated and diverse, based on the three points mentioned above, the functions are evolving and changing, showing an obvious dynamics. Structures and landscapes are outcome of man power, and human being go to build with certain purposes when facing the nature and social relations, therefore, the functions whether for structures or landscapes are all impersonal and not disappearing. Yet the concepts of functions are limited and also wide and deep, which is a unity of limit and un-limit, during the landscape design, we should insist on the purpose and compliance for the functions and not be lost in functionalism.

Though the analysis of philosophy has arguments on the usefulness of the value existing in things, Descartes philosophy mentioned "I think, therefore, I am" which idealistically stressed that things are existing widely and going before usefulness, which may be not useful. If we discuss the function and existence from philosophy, function and form, good and bad for functionalism, it will be endless, even the argument written in a great length, you cannot get a satisfactory answer. This author prefers Hegel's dialectical philosophy which raised "What is rational is actual and what is actual is rational" to make a point, and functions have never disappeared the development of landscape, the landscape is a subject strong in practice and integrity, we have to carefully consider the functions which are existing rationally. In the teaching of landscape design, we cannot cancel the functional division diagrams rashly, instead, we should first stress to satisfy and meet the functional activities is an important for the landscape design, then study the rules of functional activities of the people in the space from sociology and psychology, finally arrange factors and spaces to meet the requirements. Jan Gehl, a Danish architect adopted social psychology method in his works "Communication and Space", where, the activities of the people in public spaces are divided into three types, namely necessary activities, selective activities and social activities, and raised design methods from "promoting the communication between the people", of which, boundary effect[5] was mentioned. The boundary effect means that the people like to stay at the border of buildings or what to lean against but not in the middle, which indicate an orientation for the functional division discussion. First, urban public squares should provide functions as shade, rest, talk, look and so on; second, the functional units shall be at borders of buildings, short walls and trees. So the functions of landscapes are dynamic and evolve in an open 3D space and time, therefore, the landscape design shall have rational functional analysis and arrangement but not mechanical division for settlement. As for the "bubble diagram" and "color piece fill" mentioned above, they are just a way to express via diagrams, there is no good or bad, and the key points are the function, structural significance and mutual relations hidden in the bubbles or color pieces.

\section{REFERENCES}

[1] Tian Yinsheng, Zhang Yinger, Recognition of Functionalism and Changes of Architecture Design Thought Modes [J]. New Architecture, 1997,(02):33-34.

[2] Han Dongqing, Discussion on Dynamic Characteristics of Architectural Functions [J]. Architectural Journal, 1996,(04):34-37.

[3] Jane Jacobs, The Death and Life of Great American Cities [M]. Yilin Press, 2006,8(02).

[4] Wang Juyuan, Landscape Gardening [A]. Chinese Society of Landscape Architecture. Collected Papers of History and Development of Landscape Gardening Subject [C]. Chinese Society of Landscape Architecture, Apr 2016.

[5] Jan Gehl, Communication and Space [M]. China Architecture \& Building Press, Oct 2002. 